\title{
Microwave to gamma-ray properties of Fermi-LAT detected AGNs
}

\author{
Elisabetta Cavazzuti* \\ Italian Space Agency (ASI), Italy \\ Agenzia Spaziale Italiana (ASI) Science Data Center, I-00044 Frascati (Roma), Italy \\ E-mail: elisabetta.cavazzutilasi.it
}

\section{Dario Gasparrini}

Agenzia Spaziale Italiana (ASI) Science Data Center, I-00044 Frascati (Roma), Italy

E-mail: dario.gasparrinilasdc.asi.it

\section{Paolo Giommi}

Italian Space Agency (ASI), Italy

E-mail: paolo.giommi@asi.it

\section{Carlotta Pittori}

Agenzia Spaziale Italiana (ASI) Science Data Center, I-00044 Frascati (Roma), Italy

E-mail: carlotta.pittoridasdc.asi.it

\section{Sergio Colafrancesco}

Agenzia Spaziale Italiana (ASI) Science Data Center, I-00044 Frascati (Roma), Italy

E-mail: sergio.colafrancescolasdc.asi.it

\begin{abstract}
Blazars are a small fraction of all extragalactic sources but, unlike other objects, they are strong emitters across the entire electromagnetic spectrum. Recent data in the microwave region of the electromagnetic spectrum have become available to allow for systematic studies of blazars over large cosmological volumes. This frequency band is indeed particularly suited for the selection of blazars since at these frequencies the contamination from radio extended components with steep spectra is no longer present and the emission from the accretion process is negligible. During the first 3 months of scientific operations Fermi-LAT detected 106 bright, high-galactic latitude ( $|\mathrm{b}|>10 \mathrm{deg}$ ) AGNs with high significance. In this study we investigate the possible relations between the microwave and the gamma-ray emissions for Fermi-LAT detected AGNs belonging to WMAP 5th year bright source catalog.
\end{abstract}

The Extreme sky: Sampling the Universe above $10 \mathrm{keV}$

October 13-17 2009

Otranto (Lecce) Italy

\footnotetext{
${ }^{*}$ Speaker.
} 


\section{Introduction}

Blazars are rare extragalactic objects as they are a subset of radio loud QSOs, which in turn are only $\approx 10 \%$ of radio-quiet QSOs and Seyfert galaxies that are found in large numbers at optical and at X-ray frequencies. Despite that, the strong emission at all wavelengths that characterizes blazars, makes them the dominant type of extragalactic sources in those energy windows where the accretion onto a supermassive black hole, or other thermal mechanisms, do not produce significant radiation. For instance, in the microwave band, [Giommi 2009] showed that blazars are the largest population of extragalactic objects (see also [Toffolatti 1998]). The same is true in the $\gamma$-ray band [Abdo 2009b] and at $\mathrm{TeV}$ energies where BL Lac objects are the most frequent type of sources found in the high Galactic latitude sky.

Blazars have been known and studied in different energy windows for over 40 years, however, many questions still remain open about their physics and demographics, e.g., how are jets made and how are they accelerated? How are the relativistic particles accelerated and why is the maximum acceleration so much higher in BL Lacs [Ghisellini \& Maraschi 1989]? What are the mechanisms producing blazar variability and what is the blazar duty-cycle [Vercellone 2004], [Pittori 2007]?

We investigate these problematics using a multi-wavelength approach through WMAP $(\mu$ wave) and Fermi ( $\gamma$-ray) observations.

Primordial photons are redshifted to $\mu$-wave frequencies due to the Universe expansion and we see these photons as cosmic background in $\mu$-wave band. Tiny inhomogeneities in the early universe left their imprint on the Cosmic Microwave Background (hereafter CMB, [Penzias \& Wilson 1965]) in the form of small anisotropies in its temperature. These anisotropies contain information about basic cosmological parameters (e.g. total energy density and curvature of the universe). The ability of the present and upcoming $\mathrm{CMB}$ experiments to determine the cosmological parameters requires a careful cleaning of the CMB maps from the Galactic and extra-galactic foregrounds . This cleaning produces bright source catalogs useful to our scopes.

The microwave frequency range is likely the best region of the electromagnetic spectrum to pursue statistical studies of blazars since it is least affected by the superposition of spectral components of different nature like e.g. steep radio emission from the extended part of the jet, non-nuclear optical emission from the host galaxy or optical/UV and soft X-ray emission from an accretion disk.

Given a classification based on the synchrotron peak position of blazars $\left(v_{\text {peak }}^{\mathrm{S}}\right)$, we define lowsynchrotron-peaked, or LSP, blazar a source with $v_{\text {peak }}^{\mathrm{S}}<10^{14} \mathrm{~Hz}$, an intermediate-synchrotronpeaked, or ISP, blazar when $10^{14} \mathrm{~Hz}<v_{\text {peak }}^{\mathrm{S}}<10^{15} \mathrm{~Hz}$, and a high-synchrotron-peaked, or HSP, blazar when $v_{\text {peak }}^{\mathrm{S}}>10^{15} \mathrm{~Hz}$ ). High Synchrotron Peaked BL Lac broad band emission is currently explained with a simple homogeneous, one-zone, Synchrotron Self Compton (SSC) however more complex models involving External Compton Radiation or multiple SSC components are required to reproduce the overall Spectral Energy Distribution and the observed spectral variability of FSRQ and Low Synchrotron Peaked BL Lac. We have already studied a $\mu$-wave selected blazar sample and its X-ray properties [Giommi 2007] and verified that the $\mu$-wave flux is tightly correlated with the $\mathrm{X}$-ray one. In this project we would like to investigate a possible relation among $\mu$-wave and $\gamma$-ray fluxes.

Our strategy consists in building a complete and flux limited sample of blazars selected in the $\mu$-wave band, starting from the WMAP bright source catalogues periodically released by the 
WMAP Team. We study the properties of this $\mu$-wave selected sample of jet-dominated AGN looking for synchrotron peak distribution of the sources, $\gamma$-ray spectral indices, possible relations between $\mu$-wave fluxes and X-ray, $\gamma$-ray duty cycle, $\alpha_{\mu \gamma}$. As in [Pittori 2007], we take into account the constraints from the observed extragalactic gamma-ray background on the maximum duty cycle allowed for the selected sample of WMAP Blazars.

\section{Instruments}

The Wilkinson Microwave Anisotropic Prope (WMAP) [Bennet 2003] satellite provides an all-sky survey of the millimeter-wave sky. Its catalogues of foreground point-like sources are valuable for our study of flat-spectrum radio sources. In this work we use the 390 sources in the five-year catalogue (WMAP5) [Wright 2009]. We define the complete sample as including all sources in the WMAP5 catalogue with flux at $41 \mathrm{GHz}$ larger than $0.9 \mathrm{Jy}$. We define a flux limited sample using the source fluxes as observed in the WMAP $41 \mathrm{GHz}$ channel to have a compromise between sensitivity, completeness and the need to use a high frequency band, to avoid the steep radio components. High frequency channels in WMAP survey $(61,94 \mathrm{GHz})$ have less objects and are less deep in flux with respect to lower frequencies $(23,33,41 \mathrm{GHz})$.

The Fermi Gamma-ray Space Telescope is an international mission dedicated to the study of $\gamma$-ray emission in the Universe. The LAT, the primary instrument, is an imaging, wide field-of-view telescope, covering the energy range from below $20 \mathrm{MeV}$ to more than $300 \mathrm{GeV}$ with a sensitivity that exceeds EGRET by a factor of 30 or more. Fermi satellite commonly observes the sky in scanning mode: on a given orbit, the LAT will sweep the sky 35 degree away from the orbital plane, covering $75 \%$ of the sky. At the end of the orbit, Fermi will rock to 35 degree on the other side of the orbital. Therefore the entire sky is covered every three hours providing an uniform sky coverage within few orbit [Atwood 2009]. Thanks to the improved instrument performance (point spread function, effective area, large field of view, broad energy range) blazar observations with Fermi LAT are advancing our understanding of blazars and AGNs.

\section{WMAP 5 year and Fermi samples}

As in [Giommi 2009], we have carried out an extensive search to identify the counterparts of all the microwave foreground sources listed in the WMAP 5-year catalogue using literature and archival data. Our results allow us to define a flux limited sample of 254 high Galactic latitude microwave sources $\left(f_{41 \mathrm{GHz}} \geq 0.9 \mathrm{Jy},\left|b_{\mathrm{II}}\right|>15^{\circ}\right)$ which is virtually complete. From the analysis of classification in this complete sample we note that there is a large dominance ( $83 \%$ ) of blazars.

Most of the WMAP blazars are Low Synchrotron Peaked blazars (LSP, mainly FSRQ, $80 \%$ of the total). This is what we expect from a sample selected in the microwave band being High Synchrotron Peaked Blazars (HSP) fainter than LSP at millimetric wavelengths.

We perform a spatial cross-correlation between the WMAP5 sample and the Fermi Bright Source List [Abdo 2009a], including also the low-significance (more than 5 sigma) sources. In this way we obtain 69 objects with known microwave emission that are detected in the early phase of Fermi data acquisition. As expected $95 \%$ of this sample are blazars, all objects well known from literature. 


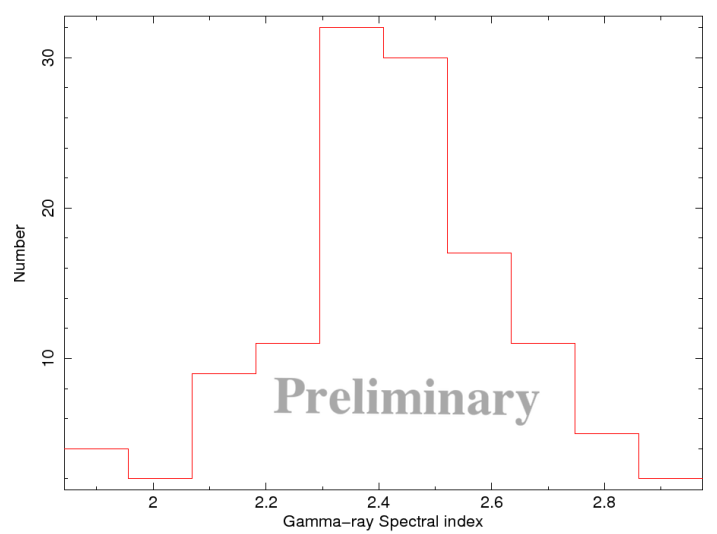

Figure 1: Photon index distribution for the WMAP-Fermi-LAT sources

We compare the distributions of $\gamma$-ray properties such as spectral index and flux: the spectral index is centered around 2.4 (fig. 1) and for most of the objects it is above 2, according to the FSRQ spectral index distribution as in [Abdo 2009b] and the dominance of FSRQs in WMAP5 sample.

As in [Pittori 2007] we define a $\mu$-wave to $\gamma$-ray slope as

$$
\alpha_{\mu \gamma}=-\frac{\log \left(f_{94 \mathrm{GHz}} / f_{100 \mathrm{MeV}}\right)}{\log \left(v_{94 \mathrm{GHz}} / v_{100 \mathrm{MeV}}\right)}
$$

and a limiting value: $\alpha_{\mu \gamma_{100 \% C G B}}=0.994$ which is the value of an hypotetical source that would produce $100 \%$ of the Cosmic Gamma-ray Background (CGB) if representative of the class.

Any source with $\alpha_{\mu \gamma}<0.994$ should have a duty cycle lower than $100 \%$ in order not to overproduce the extragalactic diffuse $\gamma$-ray background.

We estimate the blazar duty cycle by defining

$$
\text { Duty Cycle }=100 \times 10^{-11.41\left(0.994-\alpha_{\mu \gamma}\right)},
$$

where $\log \left(v_{94 \mathrm{GHz}} / v_{100 \mathrm{MeV}}\right)=11.41$.

Looking at the first 3 months of data, we have found mostly flaring blazars like EGRET and we did not found strong differences in $\alpha_{\mu \gamma}$ for the two blazar classes, FSRQ and BL Lac.

Moreover, relating the $\gamma$-ray flux $(\mathrm{E}>100 \mathrm{MeV}$ ) with the $\mu$-wave flux density at $41 \mathrm{GHz}$, apparently there is not a strong correlation (see fig. 2). However, in a recent paper [Kovalev 2009] found a correlation between high frequency radio $(15 \mathrm{GHz})$ and $\gamma$-ray fluxes. In this Kovalev's work the set of radio observations were quasi simultaneous to Fermi $\gamma$-ray fluxes. We are considering the possibility that the $\gamma$-ray high variability of blazars blurs the correlation with the $\mu$-wave band.

If we bin the microwave flux density, we can see that the fraction of Fermi-LAT detected sources increases for brightest $\mu$-wave emitters (see fig. 3). This is another hint of a relation between emission processes in microwave and $\gamma$-ray band.

\section{Conclusions}

Fermi is detecting a significant number (although a small fraction) of powerful microwave emitters. 


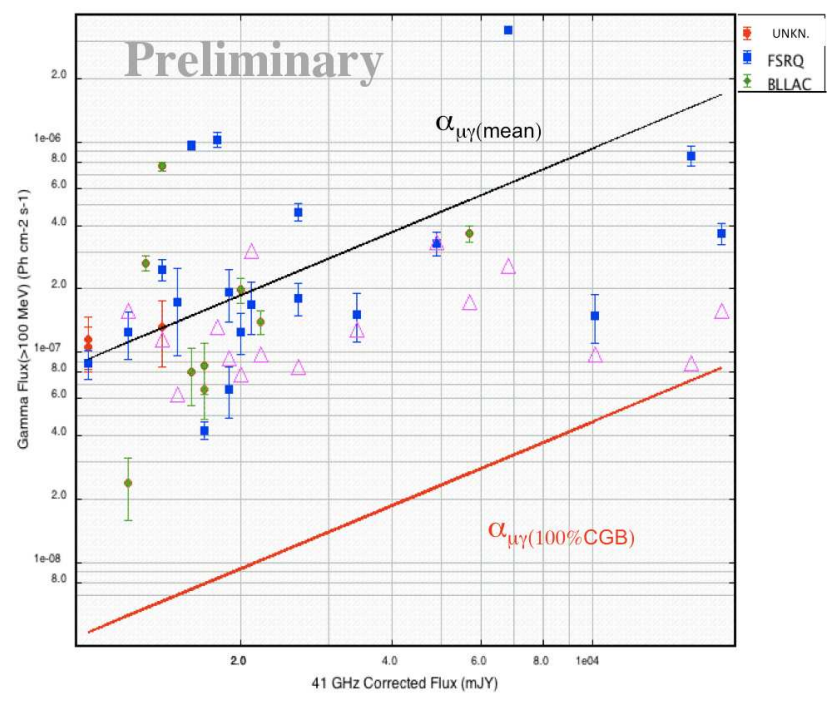

Figure 2: $\gamma$-ray Flux (>100MeV) vs $\mu$-wave flux density at $41 \mathrm{GHz}$. Blue, green and red points are FSRQ, BLLac and BZU (Blazar of optically unknown type) sources, respectively, common to both WMAP5 and Fermi BSL. Magenta triangles are previous EGRET detections, in order to show for variability. The black and red lines show the $\alpha_{\mu \gamma}$ mean and limit respectively.

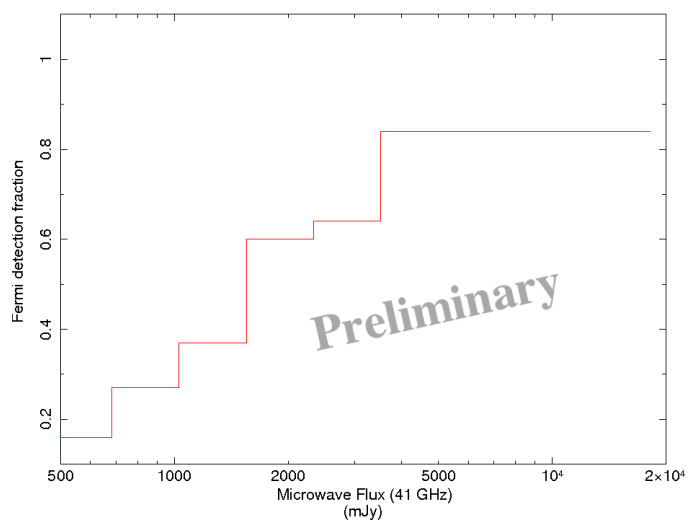

Figure 3: Fraction of the Fermi-LAT detected sources with respect to $\mu$-wave flux.

The $\mu$-wave to X-ray correlation is tight while the $\mu$-wave to $\gamma$-ray correlation is blurred by large $\gamma$-ray variability of blazars, especially with non-simultaneous data.

Planck data along with Fermi one, being both operated in survey mode, will give us unprecedented set of real simultaneous data.

The two energy bands ( $\mu$-waves and $\gamma$-rays) are indeed best suited to study blazars as their emission at these frequencies is largely dominated by non-thermal radiation. The combined study of blazars in the $\mu$-wave and in the $\gamma$-ray energy bands will probably offer a unique opportunity to understand many of the physical details of this class of black hole-dominated cosmic structures.

An hypothetical $\sim 10$ mJy LSP blazar is at the limit of the Planck sensitivity, it is detectable with a deep Swift exposure (or a less deep XMM-Newton or Chandra observation) and it is detectable by Fermi during strong flares. Since the radio $\log N-\log S$ of [Giommi 2006] predicts a space density of 5 objects per square degree with flux above $10 \mathrm{mJy}$, the Planck mission should 
detect $\approx 100,000-200,000$ blazars in the $\approx 30,000$ square degree high galactic latitude sky. A fraction of these sources will also be detected by Fermi when flaring, exactly how many strongly depends on the duty cycle.

The simultaneous observations with also Swift and other radio and optical telescopes will allow the construction of a very wide balzar database with flux measurements at radio, microwave, optical and X-ray frequencies. This extremely large sample could be used to study with great detail the statistical properties of blazars, to build the simultaneous Spectral Energy Distributions of a large and statistically well-defined sample of blazars, to study the spatial correlation function and would provide a large number of targets for future instruments operating in the still poorly explored $\mathrm{MeV}$ spectral region.

\section{References}

[Abdo 2009a] Abdo, A.A. et al, 2009a, ApJS,183, 46

[Abdo 2009b] Abdo, A.A. et al, 2009b, ApJ, 700, 597

[Atwood 2009] Atwood, W. B., et al. 2009, ApJ, 697, 1071

[Bennet 2003] Bennet, C. et al., The Astrophysical Journal, 583, 1-23 2003

[Ghisellini \& Maraschi 1989] Ghisellini, G., Maraschi, L., 1989, ApJ, 340, 181

[Giommi 2006] Giommi, P. et al. Astronomy Astrophysics, 445, 843-855, 2006

[Giommi 2009] Giommi, P., et al. 2009 A\&A, 508, 107, 2009

[Giommi 2007] Giommi, P., et al. 2007 A\&A, 468, 571, 2007

[Kovalev 2009] Y. Kovalev et al., The Astrophysical Journal Letters, 696, L17-L21, 2009

[Penzias \& Wilson 1965] Penzias, A.A., Wilson, R.W., Astrophysical Journal 142: 419-421, 1965

[Pittori 2007] Pittori, C. et al. Astrophysics and Space Science, 309, 89-94, 2007

[Toffolatti 1998] Toffolatti, L., et al. 1998, 297, 117

[Vercellone 2004] Vercellone, S. et al. 2004, MNRAS, 353, 890

[Wright 2009] Wright, E. et al., The Astrophysical Journal Supplement, 180, 283-295, 2009 